\title{
Influence of extracellular volume fraction on peak exercise oxygen pulse following thoracic radiotherapy
}

\author{
Justin M. Canada ${ }^{* *}$, Elisabeth Weiss², John D. Grizzard ${ }^{3}$, Cory R. Trankle ${ }^{1}$, Leila Rezai Gharai ${ }^{3}$, Franklin Dana ${ }^{3}$, \\ Leo F. Buckley 1,4, Salvatore Carbone ${ }^{1,5}$, Dinesh Kadariya', Anthony Ricco², Jennifer H. Jordan 1,6, \\ Ronald K. Evans ${ }^{5}$, Ryan S. Garten ${ }^{5}$, Benjamin W. Van Tassell', W. Gregory Hundley ${ }^{7}$ and Antonio Abbate ${ }^{1}$
}

\begin{abstract}
Background: Radiation-induced myocardial fibrosis increases heart failure (HF) risk and is associated with a restrictive cardiomyopathy phenotype. The myocardial extracellular volume fraction (ECVF) using contrast-enhanced cardiac magnetic resonance (CMR) quantifies the extent of fibrosis which, in severe cases, results in a noncompliant left ventricle (LV) with an inability to augment exercise stroke volume (SV). The peak exercise oxygen pulse $\left(\mathrm{O}_{2}\right.$ Pulse), a noninvasive surrogate for exercise SV, may provide mechanistic insight into cardiac reserve. The relationship between LV ECVF and $\mathrm{O}_{2}$ Pulse following thoracic radiotherapy has not been explored.

Methods: Patients who underwent thoracic radiotherapy for chest malignancies with significant incidental heart dose ( $\geq 5$ Gray (Gy), $\geq 10 \%$ heart) without a pre-cancer treatment history of HF underwent cardiopulmonary exercise testing to determine $\mathrm{O}_{2}$ Pulse, contrast-enhanced CMR, and N-terminal pro-brain natriuretic peptide (NTproBNP) measurement. Multivariable-analyses were performed to identify factors associated with $\mathrm{O}_{2}$ Pulse normalized for age/ gender/anthropometrics.

Results: Thirty patients (median [IQR] age 63 [57-67] years, 18 [60\%] female, 2.0 [0.6-3.8] years post-radiotherapy) were included. The peak $\mathrm{VO}_{2}$ was 1376 [1057-1552] $\mathrm{mL} \cdot \mathrm{min}^{-1}$, peak $\mathrm{HR}=150$ [122-164] bpm, resulting in an $\mathrm{O}_{2}$ Pulse of 9.2 [7.5-10.7] mL/beat or 82 (66-96) \% of predicted. The ECVF, LV ejection fraction, heart volume receiving $\geq 10 \mathrm{~Gy}$, and NTproBNP were independently associated with $\% \mathrm{O}_{2}$ Pulse $(P<.001)$.

Conclusions: In patients with prior radiotherapy heart exposure, \%-predicted $\mathrm{O}_{2}$ Pulse is inversely associated markers of diffuse fibrosis (ECVF), ventricular wall stress (NTproBNP), radiotherapy heart dose, and positively related to LV function. Increased LV ECVF may reflect a potential etiology of impaired LV SV reserve in patients receiving thoracic radiotherapy for chest malignancies.
\end{abstract}

Keywords: Peak exercise oxygen pulse, Extracellular volume fraction, Radiotherapy, Cardiorespiratory fitness

\footnotetext{
*Correspondence: Justin.M.Canada@vcuhealth.org

${ }^{1}$ VCU Pauley Heart Center, Department of Internal Medicine, Virginia

Commonwealth University, P.O. Box 980335, 1200 E. Broad Street,

Richmond, Virginia 23298, USA

Full list of author information is available at the end of the article
}

\section{Introduction}

Incidental cardiac radiation exposure following anticancer thoracic radiotherapy treatment increases risk of heart failure (HF) in a dose-dependent manner [1] with a predominantly restrictive cardiomyopathy phenotype characterized by diffuse fibrosis within the myocardium $[2,3]$. The restrictive cardiomyopathy phenotype original author(s) and the source, provide a link to the Creative Commons licence, and indicate if changes were made. The images or other third party material in this article are included in the article's Creative Commons licence, unless indicated otherwise in a credit line to the material. If material is not included in the article's Creative Commons licence and your intended use is not permitted by statutory regulation or exceeds the permitted use, you will need to obtain permission directly from the copyright holder. To view a copy of this licence, visit http://creativecommons.org/licenses/by/4.0/. The Creative Commons Public Domain Dedication waiver (http://creativeco mmons.org/publicdomain/zero/1.0/) applies to the data made available in this article, unless otherwise stated in a credit line to the data. 
following radiotherapy is typically a non-infiltrative disorder with endothelial cell damage resulting in microvascular dysfunction and stimulation of excessive extracellular matrix formation leading to increased myocardial fibrosis [4]. This can lead to a noncompliant left ventricle (LV) that is marked by elevated filling pressures and has limited ability to augment stroke volume (SV) [5].

The peak exercise oxygen pulse, determined with cardiopulmonary exercise testing (CPET), is the quotient of oxygen consumption $\left(\mathrm{VO}_{2}\right)$ divided by the heart rate (HR) at peak exercise. Through deduction of the Fick equation, the peak oxygen pulse can accurately serve as a noninvasive estimate of the LV SV response to exercise in both healthy subjects and HF patients [6-9]. An additional noninvasive diagnostic strategy, contrast-enhanced cardiac magnetic resonance (CMR) imaging allows tissue characterization, including quantification of the LV extracellular volume fraction (LV ECVF), a surrogate of diffuse myocardial fibrosis [10]. Knowledge of the relationship between LV ECVF and the peak exercise oxygen pulse may provide mechanistic insight into the cardiac reserve of the cancer survivor following thoracic radiotherapy. The purpose of the current study was to examine this relationship in a cross-section of patients with a history of this treatment.

\section{Methods}

This study complies with the Declaration of Helsinki, was approved by the Virginia Commonwealth University (VCU) Massey Cancer Center Protocol Review and Monitoring Committee and Institutional Review Board, and all subjects provided informed consent prior to enrollment.

\section{Patients}

Patients with a history of external-beam thoracic radiotherapy for the treatment of chest malignancies with significant incidental heart exposure defined as $\geq 5$ Gray (Gy) to $\geq 10 \%$ of the heart volume but were without a pre-cancer treatment history of overt cardiovascular disease (CVD) or HF, were prospectively enrolled. Subjects underwent CPET to determine cardiorespiratory fitness (CRF) including the peak oxygen pulse, $\mathrm{N}$-terminal probrain natriuretic peptide (NTproBNP) measurement as a marker of ventricular wall stress [11], and contrastenhanced CMR imaging. Exclusion criteria consisted of contraindications to CMR with gadolinium-contrast use, moderate or severe renal impairment (glomerular filtration rate $<60 \mathrm{~mL} / \mathrm{min} / 1.73 \mathrm{~m}^{2}$ ), pregnancy, or inability to walk on a treadmill.

Clinical variables analyzed were age, sex, race (Caucasian/African-American), history of established CVD risk factors (Yes/No; hypertension, diabetes mellitus, current cigarette smoking, hypercholesterolemia, obesity [body mass index (BMI) $\geq 30]$ ), cancer type (breast/ lung or other chest malignancy), prior chemotherapy including type and dose, presence of anemia, and current betablocker and/or angiotensin-converting enzyme inhibitor (ACEI)/ angiotensin receptor blockers (ARB) defined per medical record review and patient interview. Due to the likelihood of coexistent chronic obstructive pulmonary disease (COPD) in chest tumor patients the presence of COPD was also considered based upon Global Initiative for Chronic Obstructive Lung Disease criteria from pre-exercise spirometry [12]. Physical activity participation was quantified using a validated questionnaire [13]. Quality of life (QOL) was analyzed using the Functional Assessment of Cancer Therapy-General (FACT-G7) questionnaire [14]. Some individuals in this cohort have previously been partially characterized [15].

\section{Radiotherapy parameters}

Radiation dose calculation was performed based on a volumetric computed-tomography (CT) data set obtained during the pre-radiotherapy treatment planning session. A single radiation oncologist (E.W.) performed quantification of total radiation dose and heart volumes exposed. The heart was manually contoured on each CT-slice generating 3-dimensional structures using treatment planning software (Pinnacle, Koninklijke Philips N.V.). After radiation beam definition and target dose calculation, mean cardiac radiation dose (MCRD) was determined for the whole organ volume as well as using dose-volume histograms to generate \%volumes $(\mathrm{V})$ of the heart receiving $\geq 5,10,20,30,40$, and 50 Gray (Gy), respectively.

\section{Cardiopulmonary exercise testing}

A symptom-limited CPET was administered using a conservative ramping treadmill protocol according to established guidelines $[16,17]$. The average value for $\mathrm{VO}_{2}$ obtained during the final 30-s of exercise was used to define peak $\mathrm{VO}_{2}$. The peak exercise respiratory exchange ratio (RER) was defined as the ratio between the peak carbon dioxide production divided by the peak $\mathrm{VO}_{2}$. Predicted peak $\mathrm{VO}_{2}$ was calculated according to the reference equations proposed by Wasserman and colleagues which take into account age, gender, and anthropometric (height, bodyweight) differences [7]. The absolute peak exercise oxygen pulse (milliliters $[\mathrm{mL}]$ of $\mathrm{O}_{2}$ per heart beat) was determined by dividing the absolute peak $\mathrm{VO}_{2}$ ( $\mathrm{mL} /$ minute) by the HR at peak exercise. The predicted peak exercise oxygen pulse was calculated as the quotient of the predicted peak $\mathrm{VO}_{2}$ divided by the age-predicted maximal HR (220 - age). The peak oxygen pulse was normalized as a percent-predicted value $\left(\% \mathrm{O}_{2}\right.$ Pulse $)$ 
to account for subject differences due to age, sex, and anthropometrics. Heart rate (via 12-lead electrocardiography; GE Healthcare, Chicago, IL), blood pressure ([BP], via automated-stress BP monitor; Tango+, Morrisville, $\mathrm{NC})$, and peripheral oxygen saturation $\left(\left[\mathrm{SpO}_{2}\right]\right.$; Nellcor, Minneapolis, $\mathrm{MN}$ ) were assessed according to standard recommendations [16].

\section{Cardiac magnetic resonance}

Resting cardiac magnetic resonance imaging was performed on a Siemens Aera 1.5 Tesla scanner (Siemens Healthcare, Erlangen, Germany). All CMR parameters were interpreted by board-certified cardiovascular radiologists (J.G., L.R-G., F.D.) who were blinded to the results of the remainder of study procedures. Selected MRI sequences were obtained including cardiac dimensions, systolic function, and late-gadolinium enhancement (LGE) post-contrast images. Contrast was administered using $0.2 \mathrm{mmol} / \mathrm{kg}$ of intravenous Prohance (Bracco Diagnostics Inc., Monroe Township, NJ). The myocardial volumes; left-ventricular end-diastolic volume (LVEDV), left-ventricular end-systolic volume
(LVESV), stroke volume (SV), and cardiac output (CO) were indexed to body surface area. Myocardial tissue composition was quantified through the measurement of native $\mathrm{T} 1$ and post-contrast gadolinium-enhanced $\mathrm{T} 1$ values using a balanced steady-state free-procession modified Look-Locker inversion recovery pulse sequence. Post-contrast images were obtained at 15-min following contrast administration. Estimation of the ECVF was used to quantify diffuse myocardial injury. The ECVF was determined by the equation [18]:.

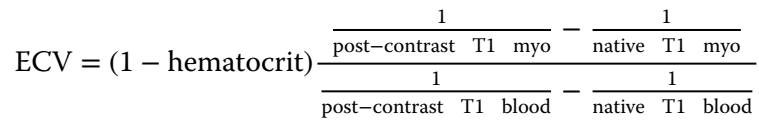

A global ECVF was determined from regions of interest in the septum on short-axis slices obtained at the base, mid, and apex of the LV myocardium. Figure 1 represents an example of an ECVF calculation. Hematocrit used in the ECVF calculations was determined non-invasively from the blood pool using a validated technique [19, 20]. Post-processing was performed using dedicated CMR analysis software (Precession, Heart Imaging Technologies, Durham, NC).

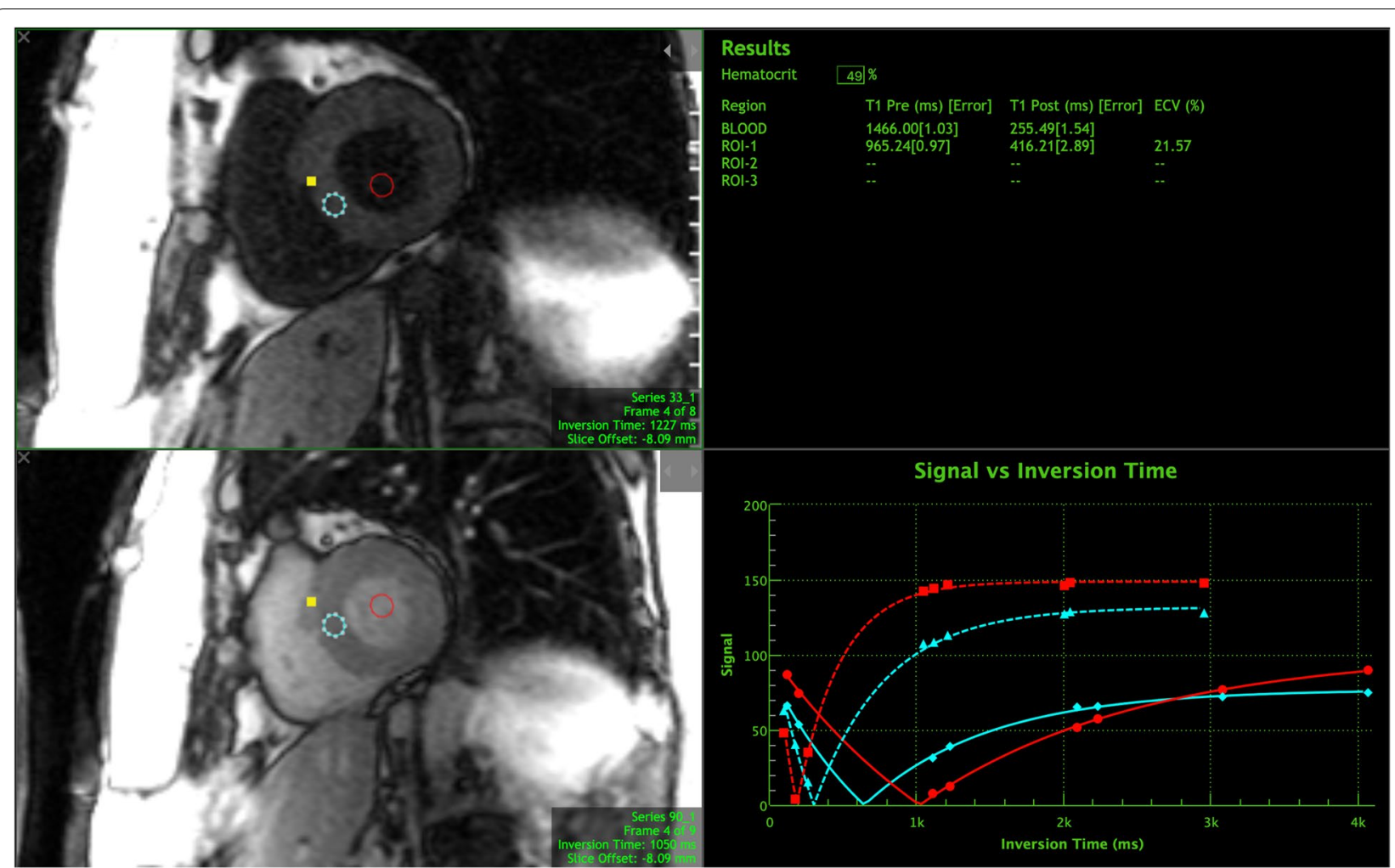

Fig. 1 Example of myocardial ECVF calculation from native T1, post-contrast T1, and blood pool inversion times. Abbreviations: $R O I=$ region of interest; $\mathrm{ECVF}=$ extracellular volume fraction; $\mathrm{ms}=$ milliseconds 


\section{Statistical analysis}

Data are reported as number (\%) or median [interquartile range] due to potential non-Gaussian distributions. Chi-square tests were performed to assess differences between nominal variables including Fisher's exact test for variables with cell count frequencies $<5$. Spearman's rank test was used for univariate analysis of associations between continuous clinical characteristics, radiotherapy, CMR, and CRF variables. The Mann-Whitney U test was performed to compare differences between groups based upon categorical variables and peak oxygen pulse values $<85 \%$ or $\geq 85 \%$ of predicted [21] and LV ECVF values $>$ or $\leq$ the median value. A threshold value of $<85 \%$ of predicted for peak oxygen pulse values has previously been shown to confer increased risk of cardiac mortality in HF patients [21]. A block multivariate linear regression model was created by first evaluating significant nominal categorical predictors of the $\% \mathrm{O}_{2}$ Pulse (block-1) followed by inclusion into a stepwise multivariate model combined with significant continuous univariate radiotherapy and $\mathrm{CMR}$ variables to determine predictors of $\% \mathrm{O}_{2}$ Pulse (block-2). All regression beta-coefficients are reported as standardized values. Collinearity diagnostics were performed on significant univariate predictors before entry into the multivariate analysis. Statistical analysis was performed using SPSS v26.0 (IBM Corp, Armonk, NY) with significance set at a $P<0.05$.

\section{Results}

\section{Patient characteristics}

Thirty patients (age 63 [57-67] years, 18 [60\%] female, 2.0 [0.6-3.8] years since completion of radiotherapy) underwent evaluation. The peak $\mathrm{VO}_{2}$ was 1376 (1057-1552) $\mathrm{mL} \cdot \mathrm{min}^{-1}$ with a peak HR of $150(122-164) \mathrm{bpm}$ resulting in a peak exercise $\mathrm{O}_{2}$ pulse of $9.2(7.5-10.7) \mathrm{mL} /$ beat that was $82(66-96) \%$ of predicted values. Table 1 provides the detailed clinical characteristics of the cohort including grouped comparisons between those with a peak exercise oxygen pulse $<85 \%$ or $\geq 85 \%$ of predicted. Sixteen (53\%) subjects demonstrated a peak exercise oxygen pulse $<85 \%$ of predicted values. When the peak exercise $\% \mathrm{O}_{2}$ Pulse was evaluated as a continuous variable according to nominal clinical characteristics there were significant differences with respect to smoking status, diabetes, obesity, cancer type, COPD, ACEI/ ARB use, and sex. There was not a significant difference between $\% \mathrm{O}_{2}$ Pulse and race, hypercholesterolemia, hypertension, anemia, beta-blocker use, or prior receipt of chemotherapy.

Table 2 provides a detailed description of selected CPET variables. Overall, the peak $\mathrm{VO}_{2}$ was moderately-reduced (62\% of predicted) compared to normalized values. The peak exercise $\% \mathrm{O}_{2}$ pulse demonstrated a significant positive correlation with the peak $\mathrm{VO}_{2}$ $(r=+0.78, P<0.001)$ and a significant inverse relationship with the cardiac biomarker NTproBNP $(r=-0.51$, $P=0.004)$. The peak $\% \mathrm{O}_{2}$ Pulse and peak $\mathrm{VO}_{2}$ were positively associated with FACT-G7 scores $(r=+0.39$, $P=0.038 ; r=+0.40, P=0.031$, respectively) reflecting higher CRF correlated with higher QOL.

The peak exercise $\% \mathrm{O}_{2}$ Pulse was not significantly associated with age, physical activity levels, rest or maximal HR or blood pressures. From a chronicity perspective, there was not a significant association between $\% \mathrm{O}_{2}$ Pulse achieved and time from cancer diagnosis or completion of chemotherapy or radiotherapy.

\section{Cardiac magnetic resonance imaging}

Cardiac magnetic resonance variables of interest are detailed in Table 3. Most patients had left-ventricular ejection fractions (LVEF) within normal range (64 [53$74] \%)$, with nearly half $(n=12[41 \%])$ of subjects exhibiting qualitative evidence of LGE with post-contrast CMR. Analysis of CMR variables with peak exercise $\% \mathrm{O}_{2}$ Pulse demonstrated a negative correlation with the composite LV ECVF $(r=-0.63, P=0.001)$, a positive correlation with LVEF ( $r=+0.55, P=0.003$ ) (Fig. 2; Panels A,B) and the resting $\mathrm{SV}$ index $(r=+0.52, P=0.011)$. There was no significant correlation between the $\% \mathrm{O}_{2}$ Pulse and native T1 $(r=-0.23, P=0.269)$, post-contrast T1 $(r=-0.20$, $P=0.340)$, or \%LGE burden $(r=-0.03, P=0.878)$. Similarly, there was no significant difference between dichotomous qualitative presence of LGE and $\% \mathrm{O}_{2}$ Pulse $(P=0.99)$.

The global LV ECVF demonstrated significant relationships with LVEF $(r=-0.51, P=0.006)$, peak $\mathrm{VO}_{2}$ $(r=-0.42, P=0.032)$, cancer type (higher in lung or other chest malignancy vs. breast cancer; $P=0.020$ ) and use of angiotensin blockade (lower in patients on ACE-I/ ARB; $P=0.007$ ) (Supplemental Fig. 1). The LV ECVF did not significantly associate with presence of established CVD risk factors (all $P>0.050$ ), MCRD or V5-50Gy heart doses (all $P>0.050)$, age $(P>0.136)$, presence of COPD $(P=0.190)$, or prior chemotherapy use $(P=0.278)$.

\section{Radiotherapy Dosimetric and chemotherapy variables}

The total prescribed radiotherapy dose was 60.0 (48.060.4) Gy, MCRD 5.6 (3.7-17.8) Gy, and the \%-volume of heart exposed to V5Gy was 39.5 (15.8-80.5)\%, V10Gy 19.3 (8.8-67.3)\%, V20Gy 7.0 (1.2-35)\%, V30Gy $2.5(0-15) \%$, V40Gy $1.0(0-7.8) \%$, and V50Gy $0(0-3) \%$, respectively. There was a negative correlation between the MCRD and the peak $\% \mathrm{O}_{2}$ Pulse $(r=-0.51, P=0.005)$. Additionally, all \%-volumes of the heart receiving between V5-V50Gy of radiotherapy demonstrated a negative association with peak $\% \mathrm{O}_{2}$ Pulse (V5Gy $[r=-0.51$, 
Table 1 Clinical Characteristics of the Cohort

\begin{tabular}{|c|c|c|c|c|}
\hline Variables & $\begin{array}{l}\text { Entire Cohort } \\
N=30\end{array}$ & $\begin{array}{l}\mathrm{O}_{2} \text { Pulse }<85 \% \\
n=16\end{array}$ & $\begin{array}{l}\mathrm{O}_{2} \text { Pulse } \geq 85 \% \\
n=14\end{array}$ & $P$-Value \\
\hline Age, y & $63[57-67]$ & $61[54-64]$ & 64 [58-69] & 0.249 \\
\hline Female & $18(60 \%)$ & $7(44 \%)$ & $10(71 \%)$ & 0.076 \\
\hline Race & & & & 0.107 \\
\hline Caucasian & $20(67 \%)$ & $9(56 \%)$ & $5(36 \%)$ & \\
\hline African-American & $10(33 \%)$ & $7(44 \%)$ & $2(14 \%)$ & \\
\hline Body Mass Index, kg/m² & $27.1[23.6-30.6]$ & $24.5[20.8-27.2]$ & $30.3[27.0-31.2]$ & 0.005 \\
\hline Body Surface Area, m² & 1.88 [1.73-1.99] & $1.81[1.71-1.94]$ & $1.92[1.79-2.14]$ & 0.132 \\
\hline COPD & $18(60 \%)$ & $13(81 \%)$ & $5(36 \%)$ & 0.023 \\
\hline FACT-G7 score & $20.0[15.0-23.5]$ & $17.5[14.3-22.3]$ & $21.0[18.0-25.0]$ & 0.041 \\
\hline Cancer type & & & & 0.008 \\
\hline Lung or Other* & $20(67 \%)$ & $14(88 \%)$ & $5(36 \%)$ & \\
\hline Breast & $10(33 \%)$ & $2(13 \%)$ & $8(57 \%)$ & \\
\hline \multicolumn{5}{|l|}{ Other Malignancies } \\
\hline Esophageal & $2(7 \%)$ & & & \\
\hline Hodgkin's Lymphoma & $1(3 \%)$ & & & \\
\hline Desmoid Tumor & $1(3 \%)$ & & & \\
\hline Castleman's Disease & $1(3 \%)$ & & & \\
\hline Time since Diagnosis, y & $2.6[1.3-4.2]$ & $2.8[1.4-7.0]$ & $2.4[0.6-2.8]$ & 0.398 \\
\hline Time since Radiotherapy, y & $2.0[0.6-3.8]$ & $2.2[0.8-5.8]$ & $2.2[0.6-2.8]$ & 0.423 \\
\hline Time since chemotherapy, y & $1.7[0.5-2.9]$ & $1.5[0.2-4.2]$ & $1.9[0.6-2.8]$ & 0.423 \\
\hline Prior Chemotherapy & $26(87 \%)$ & $14(88 \%)$ & $11(79 \%)$ & 0.617 \\
\hline \multicolumn{5}{|l|}{ CVD Risk Factors } \\
\hline Hypertension & $17(57 \%)$ & $8(50 \%)$ & $8(57 \%)$ & 0.404 \\
\hline Diabetes Mellitus & $7(23 \%)$ & $1(6 \%)$ & $6(43 \%)$ & 0.019 \\
\hline Hypercholesterolemia & $14(47 \%)$ & $8(50 \%)$ & $6(43 \%)$ & 0.566 \\
\hline Current Smoker & $6(20 \%)$ & $5(31 \%)$ & $1(7 \%)$ & 0.037 \\
\hline Obesity & $10(33 \%)$ & $3(19 \%)$ & $7(50 \%)$ & 0.064 \\
\hline Beta-blocker Use & $5(17 \%)$ & $2(13 \%)$ & $3(21 \%)$ & 0.396 \\
\hline ACEI/ARB Use & $6(20 \%)$ & $2(13 \%)$ & $4(29 \%)$ & 0.423 \\
\hline NTproBNP, pg/mL & 187 [51-310] & 298 [89-445] & 72 [31-200] & 0.013 \\
\hline
\end{tabular}

Data are listed as median and [interquartile range] or $\mathrm{n}(\%) .{ }^{*}$ Other malignancies grouped with lung cancer subjects. $P$-values are differences between groups $(<85 \%$ and $\geq 85 \%$ predicted peak exercise $\mathrm{O}_{2}$ Pulse)

Abbreviations: $y$ years, COPD chronic obstructive pulmonary disease, FACT-G7 Functional Assessment of Cancer Therapy-General (7-item version), ACEI/ARB angiotensin converting enzyme inhibitor/angiotensin receptor blocker, NTproBNP N-terminal pro-brain natriuretic peptide

$P=0.004], \quad$ V10Gy $[r=-0.56, \quad P=0.002], \quad$ V20Gy $[r=-0.45, P=0.014]$, V30Gy $[r=-0.48, P=0.009]$, V40Gy $\quad[r=-0.54, \quad P=0.003]$, V50Gy $[r=-0.42$, $P=0.023]$, respectively). Total prescribed radiotherapy dose was not associated with $\% \mathrm{O}_{2}$ Pulse $(r=-0.06$, $P=0.761$ ).

Due to the heterogeneity of chemotherapy use, comparison with peak $\% \mathrm{O}_{2}$ Pulse was restricted to those chemotherapy agents wherein at least $20 \%$ of the cohort received that agent. The doses of the chemotherapy agents analyzed included paclitaxel $(r=+0.42$, $P=0.090)$, carboplatin $(r=+0.38, \quad P=0.226)$, cyclophosphamide $(r=+0.12, P=0.774)$, and doxorubicin $(r=-0.40, P=0.313)$, which were not significantly associated with peak $\% \mathrm{O}_{2}$ Pulse. Supplemental Table 1 provides the chemotherapy regimens of the cohort.

\section{Peak oxygen pulse \& LV ECVF threshold comparisons}

When dichotomized based upon a peak oxygen pulse $<$ or $\geq 85 \%$ of predicted values, a known prognostic threshold [21], there were significant group differences between the SV index, LVEF, peak $\mathrm{VO}_{2}, \mathrm{LV}$ ECVF, and NTproBNP (Fig. 3; Tables 1-3). Furthermore, separation of the LV ECVF at the median value ( $\leq$ or $>28 \%$ ) revealed a significant difference between the LVEF and $\% \mathrm{O}_{2}$ Pulse (Fig. 3; Panels G, H). Supplemental Table 2 provides a 
Table 2 Cardiopulmonary Exercise Test Variables

\begin{tabular}{|c|c|c|c|c|}
\hline Variables & Entire Cohort & $\mathrm{O}_{2}$ Pulse $<85 \%$ & $\mathrm{O}_{2}$ Pulse $\geq 85 \%$ & $P$-Value \\
\hline Peak $\mathrm{VO}_{2}, \mathrm{~mL} \cdot \mathrm{min}^{-1}$ & 1376 [1057-1552] & 1166 [805-1330] & 1575 [1439-1946] & $<0.001$ \\
\hline Percent-predicted peak $\mathrm{VO}_{2}, \%$ & $62[52-89]$ & $53[46-61]$ & $91[69-96]$ & $<0.001$ \\
\hline Peak $\mathrm{VO}_{2}, \mathrm{~mL} \cdot \mathrm{kg}^{-1} \cdot \mathrm{min}^{-1}$ & $16.9[14.4-20.8]$ & $16.1[12.4-18.2]$ & $19.7[16.5-22.8]$ & 0.012 \\
\hline Peak $\mathrm{O}_{2}$ Pulse, $\mathrm{mL} \cdot$ beat $^{-1}$ & $9.2[7.5-10.7]$ & $7.9[6.9-9.3]$ & $10.4[9.3-12.5]$ & 0.001 \\
\hline$\% \mathrm{O}_{2}$ Pulse & $82[66-96]$ & $68[55-76]$ & $98[93-112]$ & $<0.001$ \\
\hline Peak RER & $1.02[0.95-1.09]$ & $0.97[0.92-1.09]$ & $1.05[1.00-1.11]$ & 0.045 \\
\hline Resting Heart Rate, bpm & $73[68-86]$ & $77[67-86]$ & $73[66-76]$ & 0.449 \\
\hline Exercise Heart Rate, bpm & 150 [122-164] & $136[115-157]$ & $151[141-170]$ & 0.101 \\
\hline Rest Systolic BP, mmHg & 124 [111-143] & $130[113-144]$ & 123 [103-140] & 0.531 \\
\hline Rest Diastolic BP, mmHg & $70[63-81]$ & 70 [67-82] & $68[57-79]$ & 0.374 \\
\hline Exercise Systolic BP, mmHg & 174 [155-190] & 175 [150-182] & 170 [158-201] & 0.475 \\
\hline Exercise Diastolic BP, mmHg & $70[70-80]$ & $72[70-80]$ & $70[63-81]$ & 0.329 \\
\hline Breathing Reserve, \% & $33[22-46]$ & 24 [16-44] & 37 [26-49] & 0.156 \\
\hline Peak $\mathrm{SpO}_{2}, \%$ & 97 [95-99] & 98 [94-100] & 97 [96-99] & 0.682 \\
\hline
\end{tabular}

Data are listed as median and (interquartile range). $P$-values are differences between groups ( $<85 \%$ and $\geq 85 \%$ predicted peak exercise $\mathrm{O}_{2}$ Pulse)

Abbreviations: $\mathrm{VO}_{2}$ oxygen consumption, $\mathrm{O}_{2}$ oxygen, $\% \mathrm{O}_{2}$ Pulse percent-predicted peak exercise oxygen pulse, $R E R$ respiratory exchange ratio, $B P$ blood pressure, $S p \mathrm{O}_{2}$ peripheral oxygen saturation

Table 3 Cardiac Magnetic Resonance Imaging Parameters

\begin{tabular}{lllll}
\hline Variables & $\begin{array}{l}\text { Entire Cohort } \\
\mathbf{N = 2 7}\end{array}$ & $\begin{array}{l}\text { Peak } \mathbf{O}_{\mathbf{2}} \text { Pulse }<\mathbf{8 5 \%} \\
\boldsymbol{n = 1 2}\end{array}$ & $\begin{array}{l}\text { Peak } \mathbf{O}_{\mathbf{2}} \text { Pulse } \geq \mathbf{8 5 \%} \\
\boldsymbol{n}=\mathbf{1 5}\end{array}$ \\
\hline LVEF, \% & $64[53-74]$ & $59[50-65]$ & $67[62-75]$ & $\mathbf{P}$-Value \\
LVEDV Index, $\mathrm{mL} / \mathrm{m}^{2}$ & $62[55-71]$ & $61[49-71]$ & $62[56-74]$ & 0.046 \\
LVESV Index, $\mathrm{mL} / \mathrm{m}^{2}$ & $25[18-32]$ & $24[18-38]$ & $25[15-32]$ & 0.651 \\
LV SV Index, $\mathrm{mL} / \mathrm{m}^{2}$ & $38[31-44]$ & $32[27-42]$ & $39[34-45]$ & 0.695 \\
LV Cardiac Index, L·min ${ }^{-1} / \mathrm{m}^{2}$ & $2.7[2.2-3.0]$ & $2.3[1.8-3.0]$ & $2.7[2.6-2.9]$ & 0.044 \\
Presence of LGE & $12(44 \%)$ & $5(42 \%)$ & $7(47 \%)$ & 0.190 \\
$\quad$ LGE, \% & $0[0-3]$ & $0[0-2]$ & $2[0-4]$ & 0.274 \\
Native T1-Global, ms & $1042[1013-1063]$ & $1044[1019-1070]$ & $1039[1009-1047]$ \\
Post-contrast T1-Global, ms & $434[410-463]$ & $425[386-473]$ & $436[416-454]$ & 0.427 \\
LV ECVF-Global, \% & $28[26-31]$ & $29[28-32]$ & $27[25-29]$ & 0.631 \\
\hline
\end{tabular}

Data are listed as median and [interquartile range] or $\mathrm{n}(\%)$. $P$-values are differences between groups $\left(<85 \%\right.$ and $\geq 85 \%$ predicted peak exercise $\mathrm{O}_{2}$ Pulse) Abbreviations: LVEF left-ventricular ejection fraction, LVEDV left-ventricular end-diastolic volume, LVESV left-ventricular end-systolic volume, SV stroke volume, LGE lategadolinium enhancement, T1 myocardial T1, LVECVF left-ventricular extracellular volume fraction

comparison of groups separated by the LV ECVF $\leq 28 \%$ or $>28 \%$ of the median value.

\section{Multivariate analysis model}

In the multivariate model, significant nominal-level clinical predictors of $\% \mathrm{O}_{2}$ Pulse analyzed in block-1 included: smoking status, diabetes, obesity, sex, cancer type, ACEI/ ARB use, and COPD. Cancer type was the only nominal variable retained $(\beta=.644, P<0.001)$ in block- 1 of the multivariate model with an adjusted- $R^{2}$ of 0.394 .

When evaluating radiotherapy dose parameters, collinearity diagnostics revealed significant multicollinearity between the MCRD and V5-V50Gy doses. Due to this finding, the cardiac V10Gy was the only radiotherapy variable entered into the multivariate model as it had the highest univariate relationship with $\% \mathrm{O}_{2}$ Pulse.

In block- 2 of the multivariate model, cancer type (block-1) was added to the significant univariate continuous predictors: LV ECVF, LVEF, cardiac V10Gy, and NTproBNP into a stepwise analysis. The LV ECVF $(\beta=-.281, P=0.049)$, LVEF $(\beta=.455, P=0.002)$, cardiac V10Gy $(\beta=-.330, P=0.006)$, and NTproBNP $(\beta=-.319, P=0.013)$ were retained in the model as independent predictors of $\% \mathrm{O}_{2}$ Pulse while cancer type was 

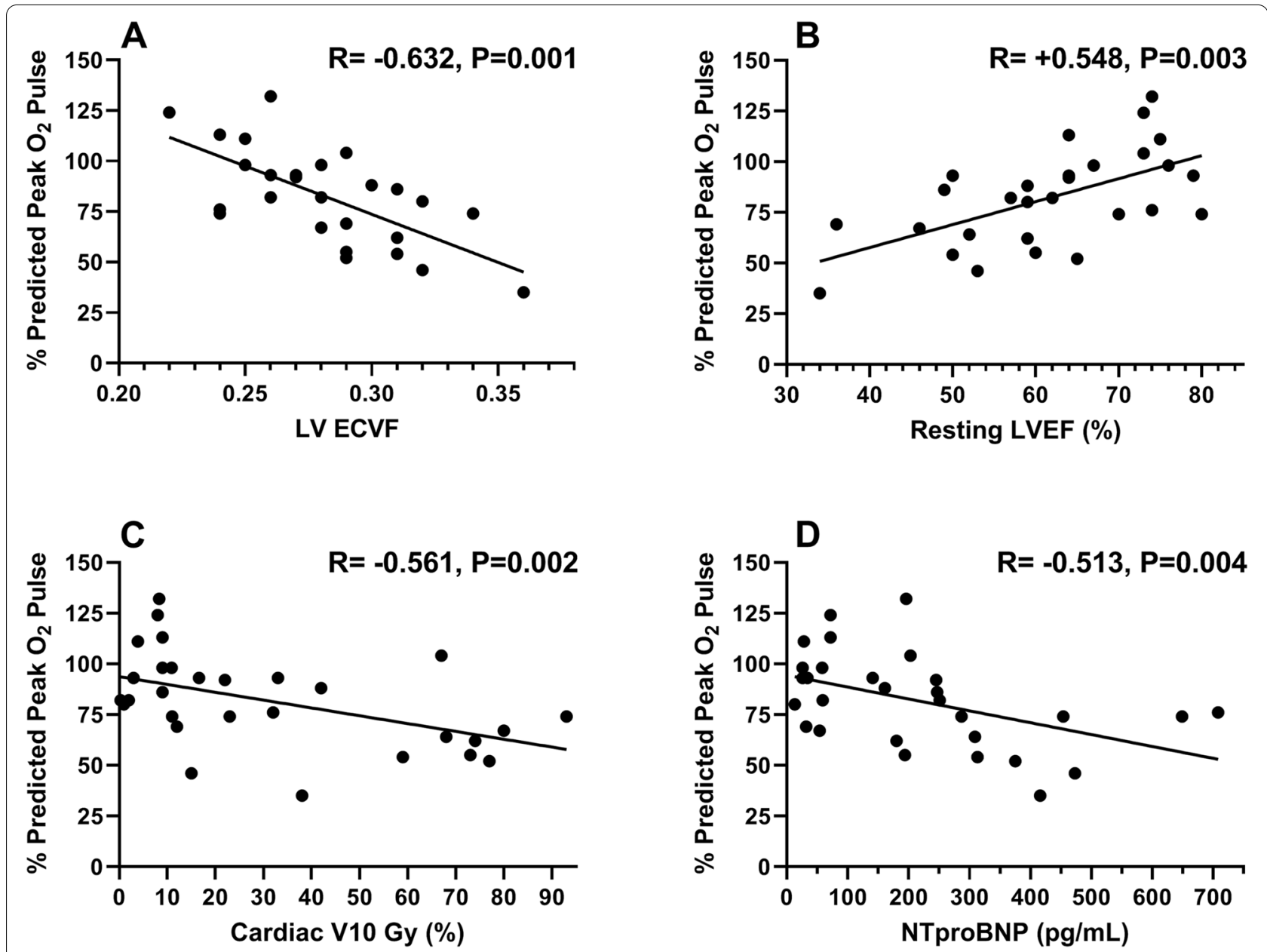

Fig. 2 Independent predictors of peak exercise $\% \mathrm{O}_{2}$ pulse. Legend: Panel A - correlation between $\mathrm{LV}$ ECVF and $\% \mathrm{O}_{2}$ pulse. Panel B - correlation between resting LVEF and $\% \mathrm{O}_{2}$ pulse. Panel C - correlation between cardiac V10Gy and $\% \mathrm{O}_{2}$ pulse. Panel $\mathbf{D}$ shows the inverse correlation between NTproBNP and $\% \mathrm{O}_{2}$ pulse. Abbreviations: $\% \mathrm{O}_{2}$ pulse $=\%$-predicted oxygen pulse; $\mathrm{ECVF}=$ extracellular volume fraction; LVEF = left-ventricular ejection fraction; $\mathrm{V} 10 \mathrm{~Gy}=\%$ volume of the heart receiving $\geq 10 \mathrm{Gray} ; \mathrm{NTproBNP}=\mathrm{N}$-terminal pro-brain natriuretic peptide

removed from the model. The overall-model fit demonstrated an adjusted- $R^{2}$ of 0.732 .

\section{Discussion}

In this cross-sectional analysis of patients with significant incidental heart exposure following thoracic radiotherapy without an established history of CVD or HF, we found peak exercise $\% \mathrm{O}_{2}$ Pulse (a normalized surrogate of exercise LV SV response) was inversely associated with a CMR-derived marker of diffuse myocardial fibrosis (LV ECVF), ventricular wall stress (NTproBNP), cardiac radiation dose, and positively related to cardiac function (LVEF). This demonstrates a potential mechanistic relationship between the effects of thoracic radiotherapy on myocardial function and tissue composition (evidenced by increased LV ECVF, decreased LVEF, elevated
NTproBNP) and its resulting influence on cardiac reserve measured via peak exercise $\% \mathrm{O}_{2}$ Pulse. Although the adverse effects of thoracic radiotherapy on late CVD/ HF risk are well-known optimal methods for screening to detect subclinical cardiac dysfunction and the role of advanced imaging or exercise testing remain understudied $[22,23]$.

We recently observed reductions in peak $\mathrm{VO}_{2}$ following thoracic radiotherapy driven primarily by impaired diastolic reserve [15, 24]. Increased interstitial myocardial fibrosis that can occur following cancer treatment can impair LV systolic and diastolic function [25]. Work has demonstrated elevated LV ECVF in anthracycline-treated patients (29\% received chest radiation) that was negatively associated with diastolic function with lower diastolic function in those with concomitant reduced systolic 

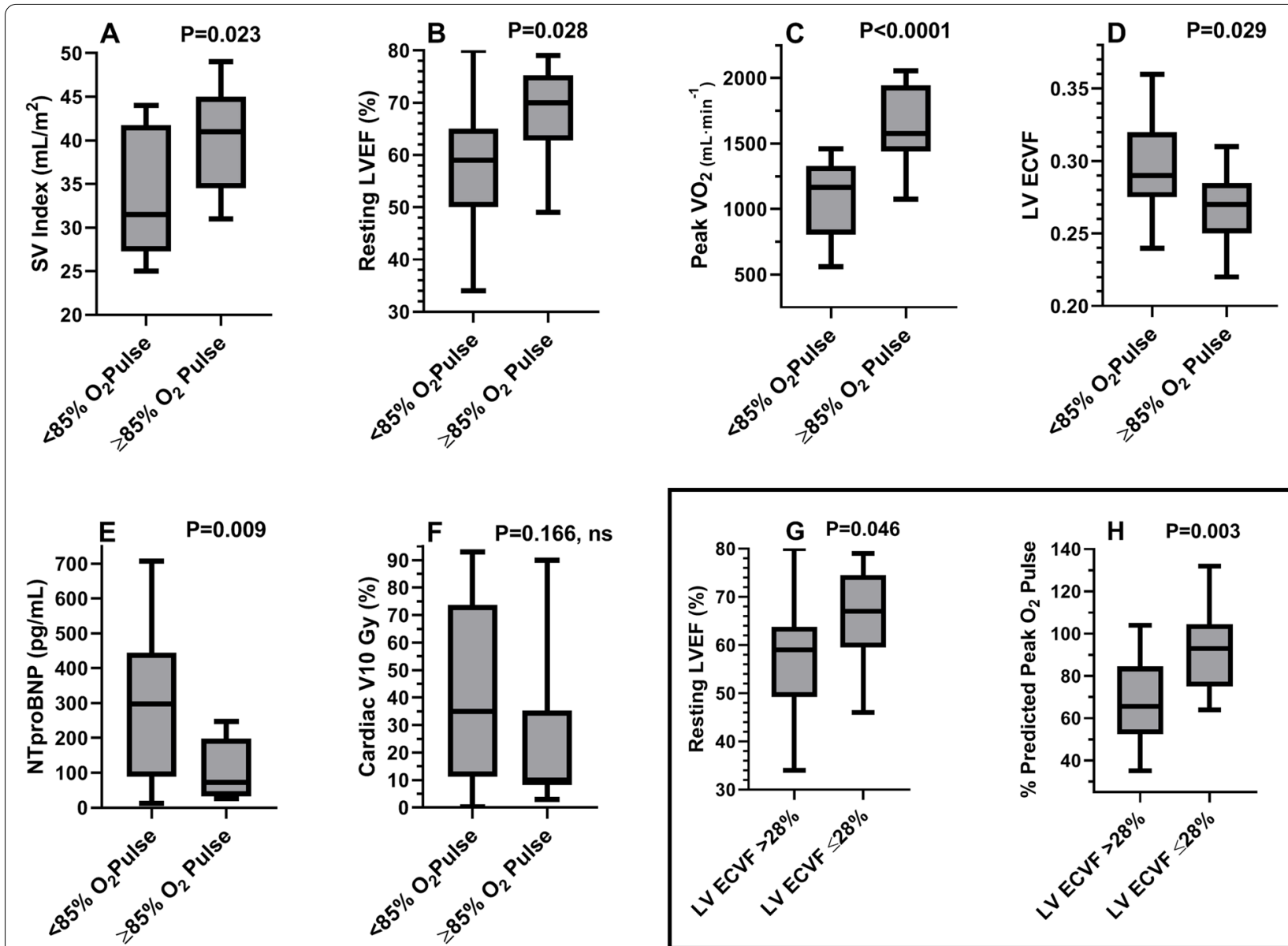

Fig. 3 Differences between groups based upon percent-predicted peak exercise $\mathrm{O}_{2}$ pulse or median LV ECVF. Legend: Box and whisker plots (Panels A-F) demonstrating median (horizontal line within rectangular box), interquartile range, whiskers, and range between groups based upon $\% \mathrm{O}_{2}$ pulse $(<$ or $\geq 85 \%)$. Panel A: Lower SVI in those with peak $\mathrm{O}_{2}$ pulse $<85 \%$ of predicted. Panel B: Lower LVEF in those with a peak $\mathrm{O}_{2}$ pulse $<85 \%$ of predicted. Panel C: Lower peak $\mathrm{VO}_{2}$ in those with a peak $\mathrm{O}_{2}$ pulse $<85 \%$ of predicted. Panel D: Higher LV ECVF in those with a peak $\mathrm{O}_{2}$ pulse $<85 \%$ of predicted. Panel E: Higher NTproBNP levels in those with a peak $\mathrm{O}_{2}$ pulse $<85 \%$ of predicted. Panel F: Trend towards higher V10Gy in those with a peak $\mathrm{O}_{2}$ pulse $<85 \%$ of predicted. Panel G: Lower resting LVEF in those with LV ECVF values $>$ median. Panel H: Lower peak $\% \mathrm{O}_{2}$ pulse values in those with LV ECVF >median. Abbreviations: $\mathrm{SVI}=$ stroke volume index; $\mathrm{O}_{2}=$ oxygen; $\mathrm{LVEF}=$ left-ventricular ejection fraction; $\mathrm{VO}_{2}=$ oxygen consumption; $\mathrm{ECVF}=$ extracellular volume fraction; $\mathrm{NTproBNP}=\mathrm{N}$-terminal pro-brain natriuretic peptide; $\mathrm{V} 10 \mathrm{~Gy}=\%$ volume of heart receiving $\geq 10$ Gray

function [26]. Takagi and colleagues prospectively evaluated changes in global LV function and myocardial tissue characterization in 24 esophageal cancer patients following chemo-radiation therapy which demonstrated early changes in LV ECVF and native T1 (increased 0.5-years after) followed by late (1.5-years after) adverse changes in the SV index [27]. Although CRF was not evaluated it highlights the ability of LV ECVF to detect early subclinical changes that translate into later functional decline (reduced SV). In childhood cancer survivors that previously underwent anthracycline-based chemotherapy regimens (17\% received chest irradiation), Tham et al. demonstrated an inverse relationship between LV ECVF and peak $\mathrm{VO}_{2}$, however, oxygen pulse was not reported [28]. Similarly, Duca et al. showed an inverse association between LV ECVF and functional status (6-min walk distance, New York Heart Association class) and stroke volume in patients with HF and preserved ejection fraction (HFpEF) [29]. In a prospective study of the ability to differentiate hypertensive heart disease and HFpEF, Mordi et al. demonstrated LV ECVF was an excellent discriminator between hypertension and HFpEF (area under the curve $=0.88$ ) and was inversely associated with peak $\mathrm{VO}_{2}$ [30].

The peak oxygen pulse is an expression of peak $\mathrm{VO}_{2}$ that by its nature corrects for $\mathrm{HR}$ and when further corrected 
for age, sex, and anthropometrics can differentiate cardiac from noncardiac causes of exercise intolerance [31, 32]. The findings of the current study have implications, as the peak $\mathrm{O}_{2}$ pulse is associated with risk for sudden cardiac death, fatal coronary heart disease, CVD, and allcause mortality [33-35]. Moreover, the peak $\mathrm{O}_{2}$ Pulse provides additive predictive accuracy to intermediaterange peak $\mathrm{VO}_{2}\left(10-14 \mathrm{~mL} \cdot \mathrm{kg}^{-1} \cdot \mathrm{min}^{-1}\right)$ for mortality risk assessment in HF patients [21]; some reports have even suggested superiority of the peak $\mathrm{O}_{2}$ pulse over peak $\mathrm{VO}_{2}$ for predicting clinical HF events particularly when it's normalized for body mass [36]. Although not reporting oxygen pulse, in a retrospective analysis of patient with cancer therapy-induced HF (CTHF) (44\% received chest radiotherapy) compared with non-cancer therapy HF (NCTHF), CTHF patients demonstrated a distinct profile of higher LVEF and worse LV diastolic and systolic (global longitudinal strain) function that was associated with a lower peak $\mathrm{VO}_{2}$ and higher incidence of the composite endpoint (all-cause mortality, heart transplant or LV assist device implant) [23]. Furthermore, in an adjusted multivariate analysis (including peak $\mathrm{VO}_{2}, \mathrm{VE} /$ $\mathrm{VCO}_{2}$ slope) CTHF was associated with a higher risk of death and the composite endpoint.

In oncological surgery patients, an abnormal exercise oxygen pulse response has been associated with increased mortality, neoadjuvant treatments, and was a strong independent predictor of post-operative cardiopulmonary complications even surpassing peak $\mathrm{VO}_{2}[37-$ 39]. Collectively, these findings suggest a potential role of CPET including measurement of oxygen pulse in the risk stratification and determination of the causes of exercise intolerance in cancer survivors including those receiving thoracic radiotherapy. To the best of our knowledge, we are not aware of any prior studies that have examined the association between the peak exercise oxygen pulse and LV ECVF.

\section{Study limitations}

The limitations of this study are its small-sample size, single-center, and cross-sectional nature, limiting the findings to hypothesis generating rather than definitively establishing causality and require further exploration. Another limitation is that evaluation of the oxygen pulse is complex, as it depends upon many factors such as fitness, body mass, and is also significantly influenced by arterial oxygen supply and peripheral oxygen extraction. However, in the current study normalization of the oxygen pulse reported as a $\%$-predicted value accounted for the influences of age, sex, and anthropometrics. Furthermore, arterial oxygen supply (quantified by $\mathrm{SpO}_{2}$ ), presence of anemia, or COPD were not significant predictors of $\% \mathrm{O}_{2}$ Pulse in the current study. Unfortunately, the influence of peripheral oxygen extraction was not directly assessed in the current study. Future studies should explore the relationships of radiotherapy dose to specific cardiac substructures with $\% \mathrm{O}_{2}$ Pulse and LV ECVF for a more precise mechanistic understanding of radiotherapyrelated cardiac dysfunction [40]. Lastly, the synthetic hematocrit used for the derivation of LV ECVF was analyzed using an automated method based on the linear relationship between the longitudinal $\mathrm{T} 1$ relaxation properties of the blood and blood hematocrit versus direct assessment.

\section{Conclusions}

A multimodality noninvasive assessment, including myocardial tissue composition (using contrast-enhanced CMR to derive LV ECVF) and assessment of cardiac reserve (using CPET to determine the normalized peak exercise $\% \mathrm{O}_{2}$ Pulse) may provide mechanistic insight into the causes of exercise intolerance in the cancer survivor following thoracic radiotherapy, suggesting that an expansion of myocardial LV ECV may be contributing to the inability to increase LVSV during exercise leading to reductions in exercise capacity. Although requiring further study, our findings indicate reductions in $\% \mathrm{O}_{2}$ Pulse may represent subclinical reductions in stroke volume that is associated with elevations in LV ECVF. This may allow early detection of radiotherapy-related myocardial dysfunction prior to the onset of overt HF thus opening the door to prophylactic therapeutic interventions.

\section{Abbreviations \\ CMR: Cardiac magnetic resonance imaging; COPD: Chronic obstructive pulmonary disease; CPET: Cardiopulmonary exercise test; CRF: Cardiorespira- tory fitness; CVD: Cardiovascular disease; CT: Computed tomography; ECVF: Extracellular volume fraction; FACT-G7: Functional Assessment of Cancer Therapy-General; Gy: Gray units; HF: Heart failure; HR: Heart rate; LGE: Late- gadolinium enhancement; LVEDV: Left-ventricular end-diastolic volume; LVEF: Left-ventricular ejection fraction; LVESV: Left-ventricular end-systolic volume; MCRD: Mean cardiac radiation dose; NTproBNP: N-terminal pro-brain natriuretic peptide; $\mathrm{O}_{2}$ : Oxygen; $\% \mathrm{O}_{2}$ Pulse: Percent-predicted peak oxygen pulse; RER: Respiratory exchange ratio; $\mathrm{SpO}_{2}$ : Peripheral oxygen saturation; SV: Stroke volume; T1:Time constant representing the recovery of longitudinal magnetization; $\mathrm{V}$ : Volume; $\mathrm{VO}_{2}$ : Oxygen consumption.}

\section{Supplementary Information}

The online version contains supplementary material available at https://doi. org/10.1186/s40959-021-00127-6.

Additional file 1.

Additional file 2.

Additional file 3. 


\section{Acknowledgements}

The authors would like to thank all of the patients and staff in the VCU Massey Cancer Center, VCU Clinical Research Unit, and VCU Pauley Heart Center who participated in this work.

\section{Authors' contributions}

All authors have approved this manuscript for publication. JDG, LRG, and FD had a role in CMR acquisition, CMR analysis, and manuscript writing; JHJ had a role in interpretation of data and manuscript writing; AR had a role in radiotherapy data acquisition, radiotherapy data analysis, and manuscript writing; CRT, SC, LFB, DK had a role in acquisition of data, and manuscript writing; RKE, $R G, B V T$ had a role in study conception, design, and analysis and interpretation of data, and manuscript writing; WGH had a role in manuscript design and revisions; JMC, EW (radiotherapy data acquisition, radiotherapy data analysis), AA had a role in study conception, design, acquisition of data, analysis and interpretation of data, and manuscript writing.

\section{Funding}

This work was supported by a Virginia Commonwealth University Massey Cancer Center Pilot Project grant \#P30CA016059K. This study was also supported by a Center for Clinical and Translational Research grant \#1UL1TR002649 from the National Center for Advancing Translational Science to Virginia Commonwealth University, Richmond, Virginia, United States.

\section{Availability of data and materials}

The dataset analyzed during the current study are available from the corresponding author upon reasonable request.

\section{Declarations}

\section{Ethics approval and consent to participate}

This research involved human subjects and was performed in accordance with the Declaration of Helsinki. All participants provided informed consent. This study was approved by the Institutional Review Board of Virginia Commonwealth University.

\section{Consent for publication}

Not applicable.

\section{Competing interests}

AA has served as a consultant for Astra Zeneca, Janssen, Merck, Novartis, Olatec, and Serpin pharma. BVT has served as a consultant for Novartis and Serpin Pharma. EW reports financial relationships through grants with Varian Medical Systems and the National Institutes of Health. SC is supported by a Career Development Award \#19CDA34660318 from the American Heart Association. The remaining authors have nothing to disclose in regard to this study and no conflicts of interest exist for all listed authors.

\section{Author details}

${ }^{1}$ VCU Pauley Heart Center, Department of Internal Medicine, Virginia Commonwealth University, P.O. Box 980335, 1200 E. Broad Street, Richmond, Virginia 23298, USA. ${ }^{2}$ Department of Radiation Oncology, Virginia Commonwealth University, Richmond, Virginia, USA. ${ }^{3}$ Department of Radiology, Virginia Commonwealth University, Richmond, Virginia, USA. ${ }^{4}$ Department of Pharmacy Services, Brigham and Women's Hospital, Boston, MA, USA. ${ }^{5}$ Department of Kinesiology \& Health Sciences, College of Humanities \& Sciences, Virginia Commonwealth University, Richmond, Virginia, USA. ${ }^{6}$ Department of Biomedical Engineering, Virginia Commonwealth University, Richmond, Virginia, USA. ${ }^{7}$ Department of Pharmacotherapy and Outcome Sciences, Virginia Commonwealth University, Richmond, Virginia, USA.

Received: 8 September 2021 Accepted: 30 November 2021 Published online: 18 January 2022

\section{References}

1. Saiki H, Petersen IA, Scott CG, Bailey KR, Dunlay SM, Finley RR, et al. Risk of heart failure with preserved ejection fraction in older women after contemporary radiotherapy for breast Cancer. Circulation. 2017;135(15):1388-96.

2. Filopei J, Frishman W. Radiation-induced heart disease. Cardiol Rev. 2012;20(4):184-8

3. Veinot JP, Edwards WD. Pathology of radiation-induced heart disease: a surgical and autopsy study of 27 cases. Hum Pathol. 1996;27(8):766-73.

4. Yarnold J, Brotons MC. Pathogenetic mechanisms in radiation fibrosis. Radiother Oncol. 2010 Oct;97(1):149-61.

5. Pereira NL, Grogan M, Dec GW. Spectrum of restrictive and infiltrative cardiomyopathies: part 1 of a 2-part series. J Am Coll Cardiol. 2018;71(10):1130-48.

6. Whipp BJ, Higgenbotham MB, Cobb FC. Estimating exercise stroke volume from asymptotic oxygen pulse in humans. J Appl Physiol. 1996;81(6):2674-9.

7. Wasserman K, Hansen JE, Sue DY, Stringer WW, Whipp BJ. Principles of exercise testing and interpretation : including pathophysiology and clinical applications. 4th ed. Philadelphia: Lippincott Williams \&Wilkins; 2005.

8. Agostoni PG, Wasserman K, Perego GB, Guazzi M, Cattadori G, Palermo $P$, et al. Non-invasive measurement of stroke volume during exercise in heart failure patients. Clin Sci (Lond). 2000;98(5):545-51.

9. Accalai E, Vignati C, Salvioni E, Pezzuto B, Contini M, Cadeddu C, et al. Non-invasive estimation of stroke volume during exercise from oxygen in heart failure patients. Eur J Prev Cardiol. 2021;28(3):280-6.

10. Kellman P, Wilson JR, Xue H, Ugander M, Arai AE. Extracellular volume fraction mapping in the myocardium, part 1: evaluation of an automated method. J Cardiovasc Magn Reson. 2012;14:63.

11. Chow SL, Maisel AS, Anand I, Bozkurt B, De Boer RA, Felker GM, et al. American Heart Association clinical pharmacology Committee of the Council on clinical cardiology, council on basic cardiovascular sciences, council on cardiovascular disease in the young, council on cardiovascular and stroke nursing, council on cardiopulmonary critical care perioperative and resuscitation, council on epidemiology and prevention, council on functional genomics and translational biology, council on quality of care, and outcomes research. Role of biomarkers for the prevention, assessment, and management of heart failure: a scientific statement from the American Heart Association. Circulation. 2017;135(22):e1054-91.

12. Vestbo J, Hurd SS, Agustí AG, Jones PW, Vogelmeier C, Anzueto A, et al. Global strategy for the diagnosis, management, and prevention of chronic obstructive pulmonary disease GOLD executive summary. Am J Respir Crit Care Med. 2013;187(4):347-65.

13. Craig C, Marshall A, Sjostrom M, Bauman A, Booth M, Ainsworth B, et al. International physical activity questionnaire: 12-country reliability and validity. Med Sci Sport Exerc. 2003;35(8):1381-95.

14. Yanez B, Pearman T, Lis CG, Beaumont JL, Cella D. The FACT-G7: a rapid version of the functional assessment of cancer therapy-general (FACTG) for monitoring symptoms and concerns in oncology practice and research. Ann Oncol. 2013 Apr;24(4):1073-8.

15. Canada JM, Trankle CR, Carbone S, Buckley LF, Medina de Chazal H, Billingsley $\mathrm{H}$, et al. Determinants of cardiorespiratory fitness following thoracic radiotherapy in lung or breast Cancer survivors. Am J Cardiol. 2020;125(6):988-96.

16. Fletcher GF, Ades PA, Kligfield P, Arena R, Balady GJ, Bittner VA, et al. Exercise standards for testing and training: a scientific statement from the American heart association. Circulation. 2013;128(8):873-934.

17. Arena R, Humphrey R, Peberdy MA, Madigan M. Predicting peak oxygen consumption during a conservative ramping protocol: implications for the heart failure population. J Cardpulm Rehabil. 2003;23(3):183-9.

18. Ugander M, Oki AJ, Hsu L-YY, Kellman P, Greiser A, Aletras AH, et al. Extracellular volume imaging by magnetic resonance imaging provides insights into overt and sub-clinical myocardial pathology. Eur Heart J. 2012;33(10):1268-78.

19. Treibel TA, Fontana M, Maestrini V, Castelletti S, Rosmini S, Simpson J, et al. Automatic measurement of the myocardial Interstitium: synthetic extracellular volume quantification without hematocrit sampling. JACC Cardiovasc Imaging. 2016;9(1):54-63.

20. Kammerlander AA, Duca F, Binder C, Aschauer S, Zotter-Tufaro C, Koschutnik $M$, et al. Extracellular volume quantification by cardiac magnetic resonance imaging without hematocrit sampling : ready for prime time? Wien Klin Wochenschr. 2018;130(5-6):190-6. 
21. Oliveira RB, Myers J, Araujo CG, Arena R, Mandic S, Bensimhon D, et al. Does peak oxygen pulse complement peak oxygen uptake in risk stratifying patients with heart failure? Am J Cardiol. 2009;104(4):554-8.

22. Raghunathan D, Khilji MI, Hassan SA, Yusuf SW. Radiation-induced cardiovascular disease. Curr Atheroscler Rep. 2017;19(5):22.

23. Nadruz W Jr, West E, Sengeløv M, Grove GLGL, Santos M, Groarke JDJD et al. Cardiovascular phenotype and prognosis of patients with heart failure induced by cancer therapy. Heart. 2019;105(1):34-41.

24. Canada JM, Thomas GK, Trankle CR, Carbone S, Billingsley H, Van Tassell BW, et al. Increased C-reactive protein is associated with the severity of thoracic radiotherapy-induced cardiomyopathy. Cardio-Oncology. 2020;6(1):2

25. Jordan JH, Todd RM, Vasu S, Hundley WG. Cardiovascular magnetic resonance in the oncology patient. JACC Cardiovasc Imaging. 2018;11(8):1150-72.

26. Neilan TG, Coelho-Filho OR, Shah RV, Feng JH, Pena-Herrera D, Mandry $D$, et al. Myocardial extracellular volume by cardiac magnetic resonance imaging in patients treated with anthracycline-based chemotherapy. Am J Cardiol. 2013;111(5):717-22.

27. Takagi H, Ota H, Umezawa R, Kimura T, Kadoya N, Higuchi S, et al. Left ventricular T1 mapping during chemotherapy-radiation therapy: serial assessment of participants with esophageal Cancer. Radiology. 2018;289(2):347-54

28. Tham EB, Haykowsky MJ, Chow K, Spavor M, Kaneko S, Khoo NS, et al. Diffuse myocardial fibrosis by T1-mapping in children with subclinical anthracycline cardiotoxicity: relationship to exercise capacity, cumulative dose and remodeling. J Cardiovasc Magn Reson. 2013;15:48.

29. Duca F, Kammerlander AA, Zotter-Tufaro C, Aschauer S, Schwaiger ML, Marzluf BA, et al. Interstitial fibrosis, functional status, and outcomes in heart failure with preserved ejection fraction: insights from a prospective cardiac magnetic resonance imaging study. Circ Cardiovasc Imaging. 2016;9(12):e005277.

30. Mordi IR, Singh S, Rudd A, Srinivasan J, Frenneaux M, Tzemos N, et al. Comprehensive echocardiographic and cardiac magnetic resonance evaluation differentiates among heart failure with preserved ejection fraction patients, hypertensive patients, and healthy control subjects. JACC Cardiovasc Imaging. 2018;11(4):577-85.

31. Ahmad MN, Yusuf SH, Ullah R, Ahmad MM, Ellis MK, Yousaf H, et al. Multivariate criteria Most accurately distinguish cardiac from noncardiac causes of dyspnea. Texas Hear Inst J. 2015;42(6):514-21.

32. Klainman E, Fink G, Lebzelter J, Krelbaumm T, Kramer MR. The relationship between left ventricular function assessed by multigated radionuclide test and cardiopulmonary exercise test in patients with ischemic heart disease. Chest. 2002;121(3):841-5.

33. Laukkanen JA, Kurl S, Salonen JT, Lakka TA, Rauramaa R. Peak oxygen pulse during exercise as a predictor for coronary heart disease and all cause death. Heart. 2006;92(9):1219-24.

34. Laukkanen JA, Araujo CGS, Kurl S, Khan H, Jae SY, Guazzi M, et al. Relative peak exercise oxygen pulse is related to sudden cardiac death, cardiovascular and all-cause mortality in middle-aged men. Eur J Prev Cardiol. 2018;25(7):772-82.

35. Oliveira RB, Myers J, Araújo CGS, Abella J, Mandic S, Froelicher V. Maximal exercise oxygen pulse as a predictor of mortality among male veterans referred for exercise testing. Eur J Prev Cardiol. 2009;16(3):358-64.

36. Lavie CJ, Milani RV, Mehra MR. Peak exercise oxygen pulse and prognosis in chronic heart failure. Am J Cardiol. 2004;93(5):588-93.

37. Mann J, Williams M, Wilson J, Yates D, Harrison A, Doherty P, et al. Exerciseinduced myocardial dysfunction detected by cardiopulmonary exercise testing is associated with increased risk of mortality in major oncological colorectal surgery. Br J Anaesth. 2020;124(4):473-9.

38. Fresard I, Licker M, Adler D, Lovis A, Robert J, Karenovics W, et al. Preoperative peak oxygen uptake in lung Cancer subjects with neoadjuvant chemotherapy: a cross-sectional study. Respir Care. 2016;61(8):1059-66.

39. Campione A, Terzi A, Bobbio M, Rosso GL, Scardovi AB, Feola M. Oxygen pulse as a predictor of cardiopulmonary events in lung resection. Asian Cardiovasc Thorac Ann. 2010;18(2):147-52.

40. Bergom C, Bradley JA, Ng AK, Samson P, Robinson C, Lopez-Mattei J, et al. Past, present, and future of radiation-induced cardiotoxicity: refinements in targeting, surveillance, and risk stratification. JACC CardioOncology. 2021;3(3):343-59

\section{Publisher's Note}

Springer Nature remains neutral with regard to jurisdictional claims in published maps and institutional affiliations.
Ready to submit your research? Choose BMC and benefit from:

- fast, convenient online submission

- thorough peer review by experienced researchers in your field

- rapid publication on acceptance

- support for research data, including large and complex data types

- gold Open Access which fosters wider collaboration and increased citations

- maximum visibility for your research: over 100M website views per year

At BMC, research is always in progress.

Learn more biomedcentral.com/submissions 